\title{
Antagonistic selection on body size and sword length in a wild population of the swordtail fish, Xiphophorus multilineatus: potential for intralocus tactical conflict
}

\author{
Melissa Liotta $^{1}$, Jessica Abbott ${ }^{2}$, Molly Morris ${ }^{1}$, and Oscar Rios-Cardenas ${ }^{3}$ \\ ${ }^{1}$ Ohio University \\ ${ }^{2}$ Lund University \\ ${ }^{3}$ Instituto de Ecología A.C.
}

January 28, 2021

\begin{abstract}
Alternative reproductive tactics (ARTs) have provided valuable insights into how sexual selection and life history tradeoffs can lead to variation within a sex. However, the possibility that tactics may constrain evolution through intralocus tactical conflict (IATC) is rarely considered. In addition, when IATC has been considered, the focus has often been on the genetic correlations between the ARTs, while evidence that the ARTs have different optima for associated traits and that at least one of the tactics is not at its optima is often missing. Here we investigate selection on three traits associated with the ARTs in the swordtail fish Xiphophorus multilineatus; body size, body shape and the sexually selected trait for which these fishes were named, sword length (elongation of the caudal fin). All three traits are tactically dimorphic, with courter males being larger, deeper bodied and having longer swords, and the sneaker males being smaller, more fusiform and having shorter swords. Using measures of reproductive success in a wild population we calculated selection differentials, linear and quadratic gradients, demonstrate that the tactics have different optima and at least one of the tactics is not at its optima for body size and sword length. Our results provide the first evidence of selection in the wild on the sword, an iconic trait for sexual selection. In addition, given the high probability that these traits are genetically correlated to some extent between the two tactics, our study suggests that IATC is constraining both body size and the sword from reaching their phenotypic optima. We discuss the importance of considering the role of IATC in the evolution of tactical dimorphism, how this conflict can be present despite tactical dimorphism, and how it is important to consider this conflict when explaining not only variation within a species but differences across species as well.
\end{abstract}

\section{Introduction}

Studies of the evolution of sexual dimorphism have led to many insights into the evolutionary mechanisms that can produce phenotypic diversity within a species (Darwin 1871, Andersson 1994, Lande 1980). Sexually antagonistic selection, different selective optima between males and females for a shared trait due to their different life histories, is a main driver in the evolution of sexual dimorphism (Rice 1984; Cox and Calsbeek 2009; Mank 2009). However, the evolution of sexual dimorphism can be constrained if the trait is genetically correlated between the sexes. Intralocus sexual conflict occurs when males and females have different optima for genotypes at a given locus (Chippindale et al. 2001; Arnqvist and Rowe 2005; Bonduriansky and Rowe 2005; Long and Rice 2007; Bonduriansky and Chenoweth 2009; Abbott et al. 2010). If unresolved, intralocus sexual conflict can generate gender load, reduction of the population's mean fitness due to the displacement of one or both of the sexes from their phenotypic optima (Bedhomme and Chippindale 2007). While research on intralocus conflict has mainly focused on the sexes, theory from intralocus sexual conflict is readily applicable to other polymorphisms. In particular within-sex polymorphisms such as Alternative Reproductive Tactics 
(ARTs), have only recently been considered in the context of intralocus conflict (Abbott and Svensson 2010; Morris et al. 2013; Buzatto et al. 2015; Pike et al. 2017; Pennell et al. 2018; Abbott et al. 2019a,b).

Alternative Reproductive Tactics (ARTs) are discrete variations in the reproductive phenotypes of members of the same sex within a species (Taborsky and Brockmann 2010). ARTs often evolve in response to strong sexual selection and involve alternate ways to gain reproductive success through suites of correlated behavioral, morphological, and life history traits (Oliveira et al. 2008). Analogous to the sexes, the optimal phenotype for a shared trait often differs between ARTs (Gross 1985; Emlen 1996; Moczek and Emlen 2000; Taborsky 2001; Engqvist and Taborsky 2016). Antagonistic selection should drive the evolution of tactical dimorphism, identified as a bimodal distribution of a trait within a sex; however, if the tactics have a shared genetic architecture then evolution toward their respective optimum can be constrained by intralocus "tactical" conflict (IATC, Morris et al. 2013). The few studies on intralocus tactical conflict have mainly focused on genetic correlations for traits between ARTs (Abbott and Svensson 2010; Buzatto et al. 2015; Pike et al. 2017; Buzatto et al. 2018), with much less work on the respective phenotypic optima for shared traits (Abbott and Gosden 2009). Studies of intralocus tactical conflict can help us to better understand how ARTs evolve, how they are maintained, and the evolutionary consequences that can arise from the loss of an ART (e.g. speciation). In addition, it will be important to consider the possibility of intralocus tactical conflict when examining the evolution of sexually selected traits across species, in particular if some of the species have alternative reproductive tactics and some do not.

In this study we investigated the potential for traits associated with the male ARTs in the High-backed Pygmy swordtail fish, Xiphophorus multilineatus (Rauchenberger et al. 1990) to be experiencing antagonistic selection. Xiphophorus multilineatus males are classified into two ARTs: larger courter males (Fig. 1A) that mature later and are behaviorally fixed (use only a courtship display to attract females); and smaller sneaker males that mature earlier and are behaviorally plastic, using courtship and sneak-chases when alone with a female, and switching almost exclusively to sneak-chases when courter males are present (Fig. 1B, Zimmerer and Kallman 1989). While the names of the ARTs in this species are based on the mating behaviors they use, there are a suite of associated traits that are tactically dimorphic, thus with a bimodal distribution, (Zimmerer and Kallman 1988; Moretz and Morris 2003; Morris et al. 2007; Bono et al. 2011; Rios-Cardenas et al. 2018; Liotta et al. 2019) including body size, which is genetically influenced by the number of copies of the Mc4r gene on the $Y$-chromosome (Lampert et al. 2010). The genetic influence on body size suggest that this is a good system in which to examine intralocus tactical conflict. Simulations have been used to demonstrate that for autosomal, $X$-linked, and $Y$-linked genetically-determined tactics, the estimated inter-tactical genetic correlations will generally be high (Abbott et al. 2019a). In addition, the presence of tactical dimorphism indicates the potential for IATC since dimorphism between the ARTs is likely evolving due to differing phenotypic optima, and thus net disruptive selection between ARTs (Morris et al. 2013). Although, as shown with sexually antagonistic selection and intralocus sexual conflict (Cox and Calsbeek 2009), the extent of dimorphism may not indicate the extent of conflict. Also, ARTs may not be dimorphic for a shared trait that is experiencing IATC, especially if the dimorphism is slow or difficult to evolve due to genetic correlations.

Using a wild population of $X$. multilineatus we quantified the strength and mode of selection acting on the ARTs for three morphological traits, allowing us to determine if the ARTs have different optima for these traits, as well as if one or both ARTs are not at their optima. Evidence for these two criteria are necessary for demonstrating intralocus tactical conflict (Morris et al. 2013). Estimating the strength of this conflict will require additional studies that measure the genetic correlation between the ARTs. The first trait we considered was body size, which has previously been shown to be tactically dimorphic in this species (Zimmerer and Kallman 1989; Rios-Cardenas et al. 2018; Liotta et al. 2019). Our measure of selection on body size will include mating success of individuals that survived to sexual maturity (Rios-Cardenas et al. 2007; Morris et al. 2010; Lyons et al. 2014), and not the selection on reaching sexual maturity earlier which increases smaller males' probability of reaching sexual maturity (Bono et al. 2011; Morris et al. 2016; Weinstein et al. 2019). Therefore, our results will allows us to tease apart the influence of sexual selection and natural selection on this trait. The second trait we examined was body shape, which 
has also been shown to be tactically dimorphic in X. multilineatus, as well as correlated with the propensity to use sneak-chase behavior within the sneaker ART (Liotta et al. 2019). The relationship between this trait and mating behaviors within the sneaker ART provides an opportunity to examine the prediction that behavioral plasticity will reduce selection on correlated morphological traits (DeWitt et al. 1998; Abbott et al. 2019b). Body shape in fishes is also well-documented predictor of swimming performance and locomotive ability (Webb 1984; Blake 2004) and could directly impact the successful execution of each ARTs' respective mating behavior, thus influencing fitness. Body shape also has the potential to function as a sexual signal, with deeper bodies increasing mating success through female mate preference and male-male competition (MacLaren et al. 2004). Finally, the third trait we examined was sword length, an extension of the ventral caudal fin rays, which has not yet been examined in relation to tactical dimorphism in $X$. multilineatus (Fig. 1A and B). This trait was first described in the green swordtail, X. helleri (Haeckel 1848), and is considered a hallmark of sexual selection (Darwin 1871) due to the classical tradeoff between female mate preference for longer swords (Basolo 1990a,b, 1998) and costs due to natural selection (Basolo and Alcaraz 2003; Hernandez-Jimenez and Rios-Cardenas 2012; Oufiero et al. 2014a,b). However, evidence for selection on this trait in the wild is surprisingly lacking. The implications for our results are discussed in relation to how intralocus conflict will influence our understanding of the evolution of tactical dimorphism, as well as the overall variation in sexually selected traits between species.

\section{Materials and Methods}

\subsection{Field Collection and lab conditions}

In November 2014, we exhaustively sampled a side stream of the Río Tambaque, San Luis Potosí, Mexico (Fig. 1C) for X. multilineatus using baited minnow traps and seine nets. The side stream sampled is blocked off from the main branch of the Tambaque by a strip of land and has only one entrance and exit. Water flows in from the main branch of the Tambaque and then passing over a shallow ledge meets back up with the main branch. It is easy for fish to enter the side stream and most likely exit out over the ledge, otherwise the stream is relatively self-contained. All adult males collected (identified by the presence of a fully developed copulatory organ, i.e., gonopodium) were photographed and fin-clipped for DNA in the field. Fin-clips were stored in $95 \%$ ethanol for later DNA extraction. Males were classified as either sneaker $(\mathrm{n}=44)$ or courter $(\mathrm{n}=36)$ males in the field using standard length (distance from tip of snout to end of caudal peduncle, Fig. 1D) and pigmentation patterns, as described by Zimmerer \& Kallman (1989). A subset of females ( $\mathrm{n}=47)$ collected in the stream were brought back to Ohio University to drop fry. Females were isolated in individual $21 \mathrm{~L}$ tanks and kept on a $12 \mathrm{~h}-12 \mathrm{~h}$ light-dark cycle. Fish were fed twice a day with Ken's Premium Spirulina Max flake (Ken's Fish Inc., Taunton, MA, USA) in the morning and newly hatched brine shrimp nauplii (Brine Shrimp Direct, Ogden, UT, USA) in the afternoon. Female Xiphophorus multilineatus can fertilize eggs from stored sperm for 7 months and can drop a brood every month (Meffe and Snelson 1989). Females' tanks were checked daily for fry and when found, they were collected, euthanized, and preserved in $95 \%$ ethanol. Forty-three out of the forty-seven females gave birth for a total of 526 fry. After females had exhausted sperm storage, they were fin-clipped for DNA, and fin-clips were stored in $95 \%$ ethanol.

\subsection{Microsatellite Genotyping}

In order to determine each males' reproductive success, we conducted a paternity analysis using five microsatellite loci, KonT38, KonD6, KonD21, KonT30, and KonD15 developed for the closely related species Xiphophorus montezumae (see Seckinger et al. 2002 for primer sequences and development). These loci have been shown to provide sufficient polymorphisms for assigning paternity in X. multilineatus (Luo et al. 2005). Genomic DNA was extracted from the tissue samples (fin-clips for adults and half of a fry's whole body) using the Qiagen DNeasy Kit (Qiagen, Hilden, Germany) following the manufacturer's protocol, with the exception that fry whole body tissue was digested overnight and adult fin tissue digested for 3 hours. Fry DNA was eluted at $50 \mu \mathrm{l}$ and adult DNA at $100 \mu \mathrm{l}$. DNA was extracted from and microsatellite loci amplified for a total of 649 fish (36 courter males, 44 sneaker males, 43 females, and 526 fry). Microsatellite loci were amplified in two multiplex PCR reactions for each sample (MIX1=KonT38, KonD6, KonD21, MIX2=KonT30, and KonD15). The forward primers were labeled with the fluorescent tags NED (KonT38, KonT30), VIC 
(KonD6), and 6-FAM (KonD21, KonD15). See Appendix for additional details. PCR products were run on a DNA Analyzer using the GeneScan 600 LIZ size standard (Applied Biosystems) at The Ohio State University Plant-Microbe Genomics Facility for fragment analysis. Alleles were scored using Geneious ver. 9.1.8 (Kearse et al. 2012, www.geneious.com).

Microsatellite loci quality was assessed using several programs. We used the GENEPOP ver. 1.0.5 R package (Rousset 2008) to calculate allele frequencies using the adult samples, whether loci deviated from Hardy-Weinberg equilibrium (HWE), linkage disequilibrium (LD) among pairs of loci, and null allele frequencies. P-values for HWE and LD were estimated using the Markov chain method with the following parameters: 1000 dememorization steps, 5000 batches, and 1000 iterations per batch for all tests. Additionally, MICRO-CHECKER ver 2.2.3 (Van Oosterhout et al. 2004) was used to check for scoring errors. We used CERVUS 3.0.7 (Kalinowski et al. 2007) to check for observed vs. expected heterozygosity and exclusion probabilities. When checking through scored genotypes we noticed mismatches between known dams and offspring. Therefore, dams who did not have at least one allele that matched any offspring and/or offspring in a female's broods that did not have at least one allele that matched their known dam were checked manually for scoring errors in Geneious. In most cases this issue occurred in individuals that were originally assigned homozygous genotypes, but upon further review a poorly amplified peak was found that was originally thought to have been a stutter peak. In 1 fry, 3 loci were not amplified, and in 14 fry one or more loci did not have an allele that could be matched to their known mother. Thus 15 fry were not included in subsequent paternity analyses, for a total of 511 out of 526 fry included.

\subsection{Paternity Analysis}

Paternity was assigned using CERVUS 3.0.7 (Kalinowski et al. 2007). CERVUS uses maximum likelihood to calculate a log likelihood ratio score (LOD), which is the likelihood that a male is the true parent of an offspring given the known genotype of the offspring's mother and the offspring's own genotype. A positive LOD score indicates that a male is more likely to be the true sire than not. The program also calculates a "delta" score or the difference in LOD scores between the two most likely candidate sires which helps in assignment when two males have a positive LOD score for an offspring. In addition, CERVUS uses simulation to estimate a maximum and minimum confidence level in assignment of the most likely sire by taking into account the number of candidate sires (total possible sires in the population both sampled and unsampled), proportion of possible sires actually sampled, completeness of loci typed, and the estimated typing error. In our CERVUS simulation we chose 0.95 and 0.80 as the maximum and minimum confidence levels respectively. Simulation parameters were as follows: 10,000 offspring, 92 candidate sires, $87 \%$ of sires sampled, $100 \%$ of loci typed, and 0.01 error rate. We estimated the number of candidate sires as the average number of males collected in the Río Tambaque over 5 sampling years and percentage of sires sampled represents the total number of males genotyped $(\mathrm{n}=80)$. Assignments with at least an $80 \%$ confidence level were kept for further analysis.

\subsection{Morphometrics and Tactical Dimorphism}

We examined three traits that are either known or suspected to be tactically dimorphic in this species: body size, body shape and sword length. We first considered widely used linear measurements of these traits (we subsequently refer to these as unidimensional traits). We measured each fish for standard length (Fig. 1D) and body depth (pelvic-fin insertion to dorsal-fin origin, Fig. 1D). Sword length was measured from the insertion point of the ventral caudal fin ray to the distal tip of the ventral ray extension (Fig. 1D) using the line tool in ImageJ ver. 1.50i (Schneider et al. 2012). Finally, we also visually confirmed that there was an extension beyond the edge of the caudal fin and that there was pigmentation along the dorsal edge of the caudal fin, given that this aspect of the sword has been identified as important in relation to female mate preference (Basolo 1995). Unless otherwise indicated, the relations between these unidimensional variables will be controlled statistically with a multiple linear regression (see Selection Analyses below).

We then used landmark-based geometric morphometrics (Zelditch et al. 2012) and principal components analyses to examine multiple components of body shape, allowing for a more detailed examination 
of how selection acts on total body shape. Males were photographed in the field using a Canon Power Shot (Canon Inc., Tokyo, Japan), plastic view box with a ruler taped to the front, and a small hand net with the netting material tightened to create a flat surface. Fish were gently pressed against one side of the view box using the net and photographed. The fish's left side was used for landmark placement, if the left side was not photographed, the right side was mirrored so that all individuals faced the same way.

We digitized seven landmarks and three semi-landmarks using tpsDig2 (Rohlf 2015) as per the methods in Liotta et al. (2019): (1) tip of rostrum; (2) forehead; (3) anterior dorsal ray insertion; (4) posterior dorsal ray insertion; (5) dorsal caudal fin insertion; (6) last scale of the midline; (7) ventral caudal fin insertion; (8) anterior insertion of the gonopodium; (9) abdomen; and (10) ventral occlusion of the operculum (Fig. 1D, Culumber et al. 2011; Johnson et al. 2014; Liotta et al. 2019). The three semilandmarks were used to characterize the forehead, total body elongation, and the abdomen (points 2, 6, and 9 , respectively). Markers were drawn for the forehead and abdomen semi-landmarks so that the points could be placed consistently by drawing a straight line between landmarks 1 and 3 and between landmarks 8 and 10 in Adobe Illustrator (Adobe Systems Inc., San Jose, CA, USA) and placing the semi-landmark $90^{\circ}$ from the midway point (Johnson et al. 2014; Liotta et al. 2019). Landmark coordinate files were obtained using tpsUtil (Rohlf 2015) and imported into R ver 3.6.0 (R Development Core Team, 2019) for analysis using the package geomorph (Adams and Otárola-Castillo 2013; Adams et al. 2018). Landmark 4 was estimated for 11 individuals and landmark 3 and 4 was estimated for 1 individual using the thin-plate spline method in the "estimate missing landmark" function in geomorph due to the dorsal fin not being extended and obscuring the view of the ray insertion. All analyses were performed in R unless stated otherwise.

As in Liotta et al. (2019), using all males (both courters and sneakers) we performed a generalized Procrustes analysis to remove information unrelated to shape (i.e. size, orientation and position) by optimal rotation of the coordinates using the least squares criterion, aligning, and scaling to unit centroid size (Rohlf and Slice 1990). Semi-landmarks were slid along their tangent directions using the Procrustes distance criterion (Rohlf 2015). The aligned coordinates were then projected into tangent space to obtain Kendall's tangent space coordinates (Rohlf 1999). These shape variables were subjected to a principal component analysis (PCA) using the covariance matrix. All axes that explained at least $10 \%$ of the variation were included in further analyses. Centroid size was calculated as the square root of the summed squared distances of each individual landmark to the centroid of the shape (Zelditch et al. 2012).

We determined if the traits standard length, body depth, sword length, sword index (calculated as sword length/standard length to correct for body size), centroid size, and body shape (PC axes 1-4) were tactically dimorphic using a Welch's two-sample independent t-test. Standard length, body depth sword length and sword index were all log transformed for normality. We also considered dimorphism in sword length in relation to extension beyond the caudal fin by comparing the number of males in each ART with and without a sword extension with a Yates corrected Chi-square test. In addition, tactical dimorphism in body shape was examined by visualizing the overlap of the $95 \%$ confidence ellipses around each group's centroid for each PC axis in relation to PC1.

\subsection{Selection Analyses}

We calculated both selection differentials $(s)$ and selection gradients for two sets of traits: three unidimensional traits (body size measured as standard length, body shape measured as depth, and sword length), and then the combination of the geometric morphometric trait for body size (centroid), all PC axes explaining $>10 \%$ variation in body shape, and sword length. Sword length was included in both sets of traits, allowing us to assess the potential of a correlation in selection on this trait and the different measures of body size and body depth.

We calculated each male's absolute fitness (total offspring sired) using the estimates of reproductive success from the paternity analysis. Any male that was not assigned offspring was given an absolute fitness of zero. Relative fitness was calculated as absolute fitness divided by mean fitness across all males. Then, all independent variables were standardized to a mean of zero and unit variance (Lande and Arnold 1983). 
The selection differentials were estimated from linear models with relative fitness regressed on each trait separately, including ART and an interaction between ART and the trait. A significant overall result suggests that at least one of the ARTs is not at its optima, and a significant interaction with ART suggests the ARTs have different optima. As selection differentials include both direct and indirect selection, we determined if the selection on the trait was direct by estimating the linear selection gradients $(\beta)$ from the standardized partial regression coefficients of multiple linear regressions. The models included relative fitness as the dependent variable, the respective standardized independent variables measuring body size, body shape and sword length, a male's ART classification, and two-way interactions between each trait and ART. A significant $\beta$ coefficient suggests that significant selection detected in the differential analysis is direct, and not due to correlation with another trait in the analysis, while a significant coefficient for the quadratic gradient $\left(y_{\text {ii }}\right)$ suggests either disruptive or stabilizing selection. The linear selection gradients $(\beta$, directional selection) were estimated from multiple regressions that included only linear terms, since if the data violates multivariate normality the linear and quadratic coefficients will be correlated (Brodie 1992). The quadratic selection gradients $\left(y_{i i}\right.$, stabilizing or disruptive selection) were estimated from models that included both linear and quadratic terms (Brodie et al. 1995). Quadratic coefficients were doubled as linear models underestimate stabilizing/disruptive selection by half (Stinchcombe et al. 2008). We used marginal effects plots to visualize the significant linear and quadratic selection gradients using the Effects package (Fox and Weisburg 2018, 2019). We also calculated and reported the selection coefficients for both of the ARTs from each of the full models. For all models, if the interaction term between a trait and ART was significant, subsequent separate models were performed for each ART in order to determine if the ARTs' slope differed from zero for that trait. Relative fitness was always calculated from the whole male population.

Violation of the assumption that model residuals are normally distributed is not problematic for estimating the linear and quadratic coefficients; however, violation of this assumption is problematic for estimating statistical significance (Lande and Arnold 1983). As this was the case for our models, significance values for the selection differentials and gradients were calculated using a re-sampling procedure in which relative fitness was randomly shuffled across individuals to obtain a null distribution for each gradient where there is no relationship between trait and fitness. Thus, probabilities were the number of times (out of 9999 permutations) in which the gradient pseudo-estimate was greater than (if the original gradient was positive) or less than (if the original gradient was negative) the original estimated gradient (Lewis et al. 2011). All statistics were performed in R ver 3.6.0 (R Core Development Team, 2019) unless stated otherwise.

\section{Results}

\subsection{Microsatellite Genotyping}

After fixing dam-offspring mismatches when possible, 4 out 5 loci were within expected population genetic parameters in the adult population, i.e. within the expectations of HWE, exhibited genetic independence (no significant LD), and had low null allele frequencies. In addition, MICRO-CHECKER reported no evidence of scoring errors for all 5 loci. However, KonT38 was problematic for all population genetic parameters. KonT38 was not within HWE $(\mathrm{P}<0.001)$, it exhibited significant linkage disequilibrium with KonD15 and KonT30 ( $\mathrm{P}=0.007, \mathrm{P}=0.032$ respectively), and the estimation for a null allele frequency was considerable (0.171). Therefore, we ran subsequent paternity analyses both with and without KonT38. In the absence of KonT38 the other 4 loci again were within expected population genetic parameters.

\subsection{Paternity Analysis}

The paternity analysis run with all 5 loci assigned paternity to 334 out of 511 fry to 51 out of 80 males (28 sneaker, 23 courters) at the minimum $80 \%$ confidence level. This is lower than the expected assignment of 416 fry ( $81 \%$ assignment rate) from the simulation, although this is likely due to the null allele increasing mismatches between fry and candidate sires. Runs excluding KonT38 with four loci were only able to assign 139 fry. Dakin and Avise (2004) report that loci with a null allele frequency equal to or below 0.2 will not greatly affect exclusion probability (in our case it was 0.171). Given that a true parent may be falsely

excluded in this case, estimates of reproductive success will likely be underestimated. Therefore, we decided 
to use the run with all five loci as this is a conservative estimate of paternity. Courter males had higher reproductive success (mean \# of fry sired $\pm \mathrm{SE}, 6 \pm 1.44$ ) than sneaker males $(3 \pm 0.64)$. The mean number of fry sired among all males was $4 \pm 0.76$ fry, with a maximum of 38 fry sired (see Fig. Appendix 1 for the distribution of paternity).

\subsection{Morphometrics and Tactical Dimorphism}

Courters and sneakers were dimorphic for standard length (SL) and body depth (BD), with courters on average $11.2 \mathrm{~mm}$ longer and $4.5 \mathrm{~mm}$ deeper than sneakers (SL: $t=18.43, d f=54.56, p<0.001$, BD: $t=$ 11.18, $d f=64.35, p<0.001)$. The swords were $8.6 \mathrm{~mm}$ longer on average for courter males as compared to sneaker males $(t=12.94, d f=61.27, p<0.001)$. The sword index, sword length relative to standard length, was also dimorphic, with courter males on average having a $39.71 \%$ longer sword per body size than sneaker males $(t=6.99, d f=57.57, p<0.001)$. The swords were also tactically dimorphic in the extent to which they extended beyond the caudal fin: there were more adults with swords that did not extend beyond the caudal fin for sneaker males (30 of 44) than for courter males (0 of 36; Yates Chi-square 36.4, $p=<$ 0.0001). Swords continue to grow after sexual maturity (Basolo 1990b), and ideally to compare sword length within and across ARTs, models should include total male age as well as size. In wild caught males it is only possible to assess age up until sexual maturity (using otolith rings) and therefore we were unable to include total age as a fixed effect in our models. However, assessment of sword length is still informative given we sampled exhaustively for males in this population which should yield a random distribution of ages.

Courters and sneakers were tactically dimorphic for centroid size, with courter males being $35.23 \%$ larger than sneaker males $(t=15.93, d f=44.37, p<0.001)$. As for body shape, the first four PC axes each explained at least $10 \%$ of the variation $(\mathrm{PC} 1=28.99 \%, \mathrm{PC} 2=22.80 \%, \mathrm{PC} 3=14.81 \%, \mathrm{PC} 4=11.40 \%)$. Courters and sneakers are dimorphic along the first major axis of variation $\mathrm{PC} 1(\mathrm{t}=10.31, \mathrm{df}=75.25, p$ $<0.001$ ), with courter and sneaker males occupying separate areas of morphospace, with no overlap in the 95\% confidence ellipses along this axis (Fig. 2). Wire-frame models generated for each PC axis represent the extreme minimum and maximum shape changes along each axis, and for PC1 suggest sneaker males were overall more fusiform than courter males which were comparatively more dorsoventrally expanded in their body shape, consistent with findings from Liotta et al. (2019). The ARTs are also dimorphic along PC2 $(\mathrm{t}=-2.68, \mathrm{df}=77.64, p=0.009)$ which described the variation in the distance between the anterior and posterior dorsal fin insertion points, the slope of the forehead (steep or shallow), deepening in the abdomen, and slight elongation or shortening of the caudal peduncle (Fig. 2). However, courters and sneakers are not dimorphic along PC3 ( $\mathrm{t}=-1.15, \mathrm{df}=65.20, p=0.254)$ and $\mathrm{PC} 4(\mathrm{t}=-0.906, \mathrm{df}=77.89, p=0.368)$.

\subsection{Selection Analyses}

The differential selection coefficients for the traits male size (standard length), body depth and sword length were all significant (Table 1A). In addition, there were significant interactions with ART for all three traits (Table 1A). When males from each ART were analyzed separately, selection differentials for all three traits for courter males were significant (Table 1A), suggesting selection for larger size, deeper bodies and longer swords (Fig. 3). However, the selection differentials for sneaker males were only significant for sword length (Table 1A), suggesting selection on sneakers for shorter swords (Fig. 3). Together, the results suggest that at least one of the ARTs is not at its optima and that the ARTs have different optima for all three of the unidimensional traits. In addition, these results suggest that selection due to mating alone (what our data measured) is stronger in courter males than in sneaker males.

In the analyses of the linear $(\beta)$ and quadradic $\left(\mathrm{y}_{\mathrm{ii}}\right)$ selection gradients, $(\beta)$ coefficient was significant for standard length, but not for the quadradic $\left(\mathrm{y}_{\mathrm{ii}}\right)$ selection coefficients or interactions with ART (Table 1B-C). This suggests that selection on standard length detected from the differentials (see above) is direct. Inspection of the marginal effects plots suggests that the directional selection for this trait may be driven primarily by courters (see Fig. Appendix 2A), similar to what was detected for the differential coefficients (see above). In the analysis of body depth, the linear $(\beta)$ and quadradic ( $\left.y_{i i}\right)$ selection gradients were not significant (Table 1B-C). This could suggest that selection detected by the selection differential on this trait 
is indirect, potentially due to a correlation with standard length. Interestingly, both the linear selection gradient $(\beta)$ as well as its interaction with ART were significant for sword length (Table 1B). These results suggest that there is direct selection on sword length, and that it is not in the same direction across the ARTs. Further analysis of the ARTs separately for this trait detected significant positive selection on courter males for longer swords, and a marginally nonsignificant negative selection coefficient $(s)$ for sneaker males (Table 1B, see Fig. Appendix 2B). Finally, as indicated by the significant quadradic selection gradient $\left(\mathrm{y}_{\mathrm{ii}}\right.$ Table 1C) and the marginal effects plots, there may also be disruptive selection on the sword for both ARTs (Fig. Appendix 2C).

In our second set of analyses that considered the geometric morphometric traits, the results were similar to those that considered only the unidimensional traits. The selection differentials were significant for centroid size, the first PC axis describing body shape (PC1), and sword length (Table 2A). These results suggest that at least one of the ARTs is not at its optima for all three of these traits. Interactions between a trait and ART were significant for body shape (PC1 and PC3) and sword length, while marginally nonsignificant for centroid size (Table 2A, Fig. 4). When the ARTs were analyzed separately for body shape (PC1) we detected significant selection for deeper bodies in courter males, but not for more narrow bodies in sneaker males. For the sword, selection was for longer swords in courter males and shorter swords in sneaker males (Table 2A, Fig. 4).

Further consideration of the linear $(\beta)$ and quadratic $\left(y_{i i i}\right)$ selection gradients detected significant linear and quadratic selection on centroid size but no significant interactions with ART (Table 2B). These results suggest that the significant selection differential for centroid size is due to direct selection, that this selection is directional, but that differences between the ARTs were not detected (Fig. Appendix 3A). Examination of the marginal effects plot for the quadratic selection gradient within each ART also suggests disruptive selection on centroid size for both ARTS, but again, differences between the ARTs were not detected (see Fig. Appendix 3B). As for body shape described by PC1, the linear selection gradient was not significant, which suggests that the selection detected on this trait with the differential may be indirect. As with the unidimensional measurement of body shape (depth), PC1 may be correlated with body size. The significant linear selection gradients $(\beta)$ as well as interactions with ART for body shape as described by axes PC2 and PC3 are interesting, given that only PC2 is tactically dimorphic (see above). Inspection of the marginal effects plots suggest that selection is in the opposite direction for the ARTs on these components of body shape (Fig. Appendix 3C and D). Further study of the potential function of the variation in these components of body shape, and their ability to evolve differences in relation to the ARTs, would be interesting to examine. Finally, we detected significant linear selection gradient $(\beta)$ on sword length as well as an interaction with ART (Table 2B), similar to the results for this trait when it was analyzed with the other unidimensional traits. Therefore, selection on the sword appears to be direct, regardless of the measures used for body size and body shape in the analyses. In addition, analyses of the ARTs separately detected selection for longer swords in courter males and shorter swords in sneaker males (Table 2B, Fig. Appendix 3E).

\section{Discussion}

Alternative Reproductive Tactics (ARTs), which involve the use of alternative mating behaviors as well as a suite of other traits, evolve due to intense intrasexual competition that imposes strong sexual selection (Oliveria et al. 2008). If selection can optimize reproductive success by alternative suites of traits, then we expect tactical dimorphism to evolve (Gross 1985; Moczek and Emlen 2000; Radwan and Klimas 2001; Sato et al. 2004). However, little is known about whether the evolutionary constraints due to intralocus tactical conflict (IATC) can prevent traits reaching their optima within an ART. Our results suggest that body size and sword length, both tactically dimorphic traits in Xiphophorus multilineatus, have different optima, and one or more of the ARTs is not at its optima for both traits, providing strong evidence of the potential for Intralocus Tactical Conflict. Body shape was also experiencing selection that was different between the ARTs, but our data did not allow us to demonstrate that the selection was direct. Finally, the selection we measured appears to be stronger for courter males than sneaker males for all of the traits except for sword length. We discuss the implications of our results in relation to the selection of these traits in this system, 
as well as the implications for considering intralocus tactical conflict in relation to the evolution of ARTs in general below.

Body size is the morphological trait that has been studied most extensively in relation to the ARTS in $X$. multilineatus. The ARTS are dimorphic for body size (Liotta et al. 2019, current study), and yet our results suggest that intralocus tactical conflict could be constraining the courter males from reaching their optimal size. Variation in body size has a strong genetic influence due to variation in both alleles and copy number of the $M c 4 r$ gene on the $Y$ chromosome (Lampert et al. 2010). However, for a trait like male body size, it is unlikely that variation in the $M c 4 r$ gene is explaining all of the variation, such that numerous autosomal genes could lead to IATC. The genetic correlation across the ARTs for this trait is currently being examined and will allow us to determine the extent to which IATC is constraining this trait. It is also interesting to note that because the selection we measured did not account for the invisible fraction (males that did not survive to reproduce, Grafen 1988) the antagonistic selection on body size we measured is not due to the differences between the ARTs in relation to the benefits and costs of reaching sexual maturity earlier (Rios-Cardenas et al. 2018, Weinstein et al. 2019). Instead, what we measured is due to influence of sexual selection alone. This is important as it suggests that intralocus tactical conflict is an additional evolutionary mechanism beyond survival costs of maturing later that can limit the evolution of this influential trait. Finally, when body size was measured as centroid size we detected disruptive selection, selection that was not different across the ARTs. Further examination of female mate preference functions as well as male-male interactions has the potential to reveal a more complex role for centroid size in this system.

The results for selection on body shape were not as clear as those for body size. The selection differentials on both the unidimensional measure of body shape (body depth) and the first PC axis for the geometric morphometric measure (PC1) were significant, but the selection gradients for both measures of this trait were marginally nonsignificant. One possible explanation is that the selection on this aspect of body shape is indirect, due to correlation with another trait. We have shown previously that body size and shape are phenotypically correlated across both courters and sneakers (Liotta et al. 2019). However, we also presented two theoretical juvenile development pathways for body shape (Liotta et al. 2019, Fig.5) that could both explain this correlation. In one, body shape differences between the ARTs is attributable to differences in ages at sexual maturity alone. In the other theoretical pathway, the differences between the ARTs in body shape are due to different developmental paths, in which case body shape could potentially evolve independently of size. To tease apart these two scenarios, future studies of the developmental trajectories of both body shape and body size across the ARTs are planned.

Another interesting result from the analyses of selection on body shape is that similar to the results for body size, the selection differentials for body depth and PC1 were significant for courter males but not sneaker males. Given that deeper bodies increased the size of the signal to female sail-fin mollies (MacLaren et al. 2004), selection for deeper bodied courter males in X. multilineatus is not too surprising, as this trait could have increased mate attraction. We had, however, expected selection for more fusiform shapes in the sneaker males to allow for less drag and improved prolonged swimming for males using the sneak-chase mating behavior. One possible explanation for the apparent lack of selection on body shape in the sneaker males is their behavioral plasticity, which could buffer selection for a body shape that is optimal for use with either behavior (Abbott et al. 2019b). The correlation between body shape and propensity to use sneak-chase we detected previously (Liotta et al. 2019) supports this interpretation. More recent work detected this same relationship in lab reared males with limited experience in addition to wild caught males, providing further evidence to suggest that the relationship is genetically influenced and not learned (Liotta et al., in prep). In addition, the fact that we could detect selection for an overall deeper body shape in the courter males, which are fixed for courtship, lends further support to this hypothesis. Finally, there is the interesting possibility of using a comparative study to further test the hypothesis that behavioral plasticity is buffering selection on body shape in this system. Two closely related species have evolutionarily lost large males size and are fixed for sneak-chase behavior (X. continens and X. pygmaeus, Ryan and Causey 1989, Morris et al. 2005). We predict that body shape in both of these species will be experiencing stronger selection than what we detected in the $X$. multilineatus sneaker males. 
Given that body shape is a multidimensional trait, and the PC axes that we examined are a composite of multiple characteristics of, in this case, body shape, they are not independent characters with specific biological meanings (Klingenberg and Monteiro 2005; Mitteroecker and Bookstein 2011; Kuchta and Svensson 2014). While this can complicate the interpretation of selection on individual PC axes, the benefit is that each axis reflects linear combinations of traits and therefore includes correlational selection between the different elements of shape described by each PC axis (Kuchta and Svensson 2014). As for direct selection on PC2, it would be interesting to further explore the potential function of a more upturned head and the elongation of the caudal peduncle. Variation in these aspects of body shape has been previously detected in relation to habitat differences (e.g. Franssen et al. 2013, Piñeros et al. 2015), but to our knowledge has not been examined in relation to mating behaviors. However, these morphological variations seem to be in accordance with the use of unsteady swimming (Langerhans 2009, Langerhans and Reznick 2010), predominantly used by courters, as opposed to a more steady swimming favored by the sneak-chase behavior used by sneakers. And finally, aspects of body shape may have aided courter males in maneuverability or even fast starts (Webb 1994; Blake 2004; Langerhans 2009; Oufiero et al. 2014a), allowing the execution of rapid back and forth turns used in both courtship display and aggressive interactions with other males.

The sword on Xiphophorus fishes has long been a trait of interest in evolutionary biology (Darwin 1871) as it illustrates the importance of trade-offs between sexual selection and natural selection (Darwin 1871, Basolo 1990a,b; Hernandez-Jimenez and Rios-Cardenas 2012; Oufiero et al. 2014b). We provide the first evidence of direct selection on this trait in a wild population, in addition to the potential for IATC to be constraining its evolution. Across many Xiphophorus species, females are known to prefer males with longer swords (Basolo 1990a,b, 1995; Rios-Cardenas and Morris 2011, although see Rosenthal et al. 2002), which would explain the direct positive selection on sword length in the courter males. Selection on the sword for sneaker males was also significant, but negative. The potential for locomotor and metabolic costs of possessing a sword (Basolo and Alcaraz 2003; Royle et al. 2006; although see Oufiero et al. 2014a) could explain the negative selection in males that primarily use coercive mating behaviors. The differences in selection on the sword across the ARTs suggests that Intralocus Tactical Conflict could be constraining the courter males from evolving longer swords, while at the same time constraining the sneaker males from evolving shorter swords. Therefore, even though sword length is dimorphic for the ARTs, neither ART is at its optima for this trait.

Further evidence that Intralocus tactical conflict may be constraining the evolution of the sword comes from comparative studies of sword length across species of Norther Swordtails fishes. There are two independent losses of the large male morphs in this clade (X. continens and X. pygmaeus, Morris et al. 2005), which would reduce or eliminate Intralocus tactical conflict. As predicted based on the selection on sword length in sneaker males we detected, loss of the larger males would reduce or eliminate Intralocus Tactical Conflict, leading to the evolution of shorter swords. In both species all males have evolutionarily lost the sword (Rauchenberger et al. 1990, Ryan and Causey 1989; Morris et al. 2005). An additional comparative study of swordtail fishes could determine if the presence of more distinct alternative mating tactics and antagonistic selection, such as detected here for the sword in $X$. multilineatus, could help explain variation in sword length across species. The sword index for $X$. multilineatus males, whether considering the average across all males $(0.4 \pm 0.02$ $\mathrm{SE}, \mathrm{N}=80)$, or for just the courter $(0.5 \pm 0.02)$, or sneaker males $(0.32 \pm 0.01)$ in the current study, is less than the average for $X$. nezahualcoyotl (0.56) and X. montezumae (1.0; lab reared males; Rauchenberger et al. 1990), species without discrete ARTs. We suggest that the evolution of this hallmark trait for sexual selection may be influenced by variation across species in intralocus tactical conflict, in addition to variation in the strength of female mate preference (Rosenthal et al. 2002) and predation (Basolo and Wagner 2004; Hernandez-Jimenez and Rios-Cardenas 2012).

Understanding tactically antagonistic selection based on data from a wild population adds to the growing body of research suggesting that IATC is constraining ARTs from reaching their fixed phenotypic optima. ARTs are ubiquitous across taxa and a better understanding of IATC will ultimately elucidate evolutionary processes and patterns we see across species with ARTs and other polymorphisms. It is also clear that IATC needs to be considered when explaining variation in sexually selected traits both within and across taxa. Finally, given that loss of an ART is associated with speciation across a range of species (ant 
species, Oettler et al. 2010; the side-blotched lizards, Corl et al. 2010; northern swordtail fishes, Morris et al. 2013), further study of how these losses release traits from intralocus tactical conflict could help explain the rapid evolution of the remaining ART (West-Eberhard 1986), which could lead to a speciation event (Bonduriansky and Chenoweth 2009; Bonduriansky 2011; Abbott et al. 2019b).

\section{References}

Abbott, J. K., S. Bedhomme, and A. K. Chippindale. 2010. Sexual conflict in wing size and shape in Drosophila melanogaster. J. Evol. Biol. 23:1989-1997.

Abbott, J. K., and T. P. Gosden. 2009. Correlated morphological and colour differences among females of the damselfly Ischnura elegans. Ecol. Entomol. 34:378-386.

Abbott, J. K., O. Rios-cardenas, and M. Morris. 2019a. Genetic correlations across genetically determined and developmentally plastic alternative reproductive tactics. bioRxiv

Abbott, J., O. Rios-Cardenas, and M. R. Morris. 2019b. Insights from intralocus tactical conflict: adaptive states, interactions with ecology and population divergence. Oikos 128:1525-1536.

Abbott, J. K., and E. I. Svensson. 2010. Morph-specific variation in intersexual genetic correlations in an intra-specific mimicry system. Evol. Ecol. Res. 12:105-118.

Adams, D. C., and E. Otárola-Castillo. 2013. Geomorph: An r package for the collection and analysis of geometric morphometric shape data. Methods Ecol. Evol. 4:393-399.

Adams, D. C., M. L. Collyer, and A. Kaliontzopoulou. 2018. Geomorph: Software for geometric morphometric analyses. $\mathrm{R}$ package version 3.0.6. https://cran.r-project.org/package=geomorph.

Andersson, M. 1994. Sexual Selection. Princeton Univ. Press, Princeton, NJ.

Arnqvist, G., and L. Rowe. 2005. Sexual conflict in nature. Pp. 1-13 in G. Arnqvist and L. Rowe, eds. Sexual conflict. Princeton University Press, Princeton.

Basolo, A. L. 1998. Evolutionary change in a receiver bias: a comparison of female preference functions. Proc. R. Soc. B Biol. Sci. 265:2223-2228.

Basolo, A. L. 1990a. Female preference for male sword length in the green swordtail, Xiphophorus helleri (Pisces: Poeciliidae). Anim. Behav. 40:332-338.

Basolo, A. L. 1990b. Female preference predates the evolution of the sword in swordtail fish. Science. 250:808810.

Basolo, A. L. 1995. Phylogenetic evidence for the role of a pre-existing bias in sexual selection. Proc. R. Soc. B Biol. Sci. 259:307-311.

Basolo, A. L., and G. Alcaraz. 2003. The turn of the sword: length increases male swimming costs in swordtails. Proc. R. Soc. B Biol. Sci. 270:1631-1636.

Basolo, A. L., and W. E. Wagner. 2004. Covariation between predation risk, body size and fin elaboration in the green swordtail, Xiphophorus helleri. Biol. J. Linn. Soc. 83:87-100.

Bedhomme, S., and A. K. Chippindale. 2007. Irreconcilable differences: when sexual dimorphism fails to resolve sexual conflict. Pp. 185-194 in D. J. Fairbairn, W. U. Blanckenhorn, T. Szekely ed. Sex, Size and Gender Roles. Oxford University Press, Oxford.

Blake, R. W. 2004. Fish functional design and swimming performance. J. Fish Biol. 65:1193-1222.

Bonduriansky, R. 2011. Sexual Selection and Conflict as Engines of Ecological Diversification. Am. Nat. 178:729-745.

Bonduriansky, R., and S. Chenoweth. 2009. Intralocus sexual conflict. Trends Ecol. Evol. 24:280-288. 
Bonduriansky, R., and L. Rowe. 2005. Intralocus sexual conflict and the genetic architecture of sexually dimorphic traits in Prochyliza xanthostoma (Diptera: Piophilidae). Evolution (N. Y). 59:1965-1975.

Bono, L. M., O. Rios-Cardenas, and M. R. Morris. 2011. Alternative life histories in Xiphophorus multilineatus: evidence for different ages at sexual maturity and growth responses in the wild. J. Fish Biol. $78: 1311-22$.

Brodie, E. D. 1992. Correlational selection for color pattern and antipredator behavior in the garter snake Thamnophis ordinoides. Evolution (N. Y). 46:1284-1298.

Brodie, E. D., A. J. Moore, and F. J. Janzen. 1995. Visualizing and quantifying natural selection. Trends Ecol. Evol. 10:313-318.

Buzatto, B. A., H. L. Clark, and J. L. Tomkins. 2018. Morph-specific artificial selection reveals a constraint on the evolution of polyphenisms. Proc. R. Soc. B Biol. Sci. 285:20180335.

Buzatto, B. A., J. S. Kotiaho, J. L. Tomkins, and L. W. Simmons. 2015. Intralocus tactical conflict: Genetic correlations between fighters and sneakers of the dung beetle Onthophagus taurus. J. Evol. Biol. 28:730-738.

Chippindale, A. K., J. R. Gibson, and W. R. Rice. 2001. Negative genetic correlation for adult fitness between sexes reveals ontogenetic conflict in Drosophila. Proc. Natl. Acad. Sci. U. S. A. 98:1671-1675.

Corl, A., A. R. Davis, S. R. Kuchta, and B. Sinervo. 2010. Selective loss of polymorphic mating types is associated with rapid phenotypic evolution during morphic speciation. Proc. Natl. Acad. Sci. U. S. A. $107: 4254-9$.

Cox, R. M., and R. Calsbeek. 2009. Sexually antagonistic selection, sexual dimorphism, and the resolution of intralocus sexual conflict. Am. Nat. 173:176-187.

Culumber, Z. W., H. S. Fisher, M. Tobler, M. Mateos, P. H. Barber, M. D. Sorenson, and G. G. Rosenthal. 2011. Replicated hybrid zones of Xiphophorus swordtails along an elevational gradient. Mol. Ecol. 20:342-356.

Dakin, E. E., and J. C. Avise. 2004. Microsatellite null alleles in parentage analysis. Heredity. 93:504-509.

Darwin, C. 1871, The Descent of Man and Selection in Relation to Sex. J Murray, London.

DeWitt, T. J., A. Sih, and D. S. Wilson. 1998. Costs and limits of phenotypic plasticity. Trends Ecol. Evol. 13:77-81.

Emlen, D. J. 1996. Artificial selection on horn length-body size allometry in the horned beetle Onthophagus acuminatus (Coleoptera: Scarabaeidae). Evolution (N. Y). 50:1219-1230.

Engqvist, L., and M. Taborsky. 2016. The evolution of genetic and conditional alternative reproductive tactics. Proc. R. Soc. B Biol. Sci. 283:2015-2945.

Fox, J., and S. Weisberg. 2018. Visualizing _t and lack of _t in complex regression models with predictor effect plots and partial residuals. Journal of Statistical Software, 87(9):1\{27, doi: 10.18637/jss.v087.i09.

Fox, J., and S. Weisberg. 2019. An R Companion to Applied Regression (3nd ed.). Thousand Oaks CA: Sage.

Franssen, N. R., J. Harris, S. R. Clark, J. F. Schaefer, L.K. Stewart. 2013. Shared and unique morphological responses of stream fishes to anthropogenic habitat alteration. Proc. R. Soc. B Biol. Sci. 280: 20122715.

Grafen, A. 1988. On the use of data on lifetime reproductive success. Pp. 454-471 in T. H. Clutton-Brock, ed. Reproductive Success. Univeristy of Chicago Press, Chicago.

Gross, M. R. 1985. Disruptive selection for alternative life histories in salmon. Nature 313:47-48.

Haeckel, J. 1848. Eine neue Gattung von Poecilien mit rochenartigem. Anklammerungs-Organe Sitzber. K. Akad. Wiss. Wien. Math. Nat. CI., 1, 289-303. 
Hernandez-Jimenez, A., and O. Rios-Cardenas. 2012. Natural versus sexual selection: Predation risk in relation to body size and sexual ornaments in the green swordtail. Anim. Behav. 84:1051-1059.

Johnson, J. B., D. C. Macedo, C. N. Passow, and G. G. Rosenthal. 2014. Sexual ornaments, body morphology, and swimming performance in naturally hybridizing swordtails (Teleostei: Xiphophorus). PLoS One 9.

Kalinowski, S. T., M. L. Taper, and T. C. Marshall. 2007. Revising how the computer program CERVUS accommodates genotyping error increases success in paternity assignment. Mol. Ecol. 16:1099-1106.

Kearse, M., R. Moir, A. Wilson, S. Stones-Havas, M. Cheung, S. Sturrock, S. Buxton, A. Cooper, S. Markowitz, C. Duran, T. Thierer, B. Ashton, P. Meintjes, and A. Drummond. 2012. Geneious Basic: An integrated and extendable desktop software platform for the organization and analysis of sequence data. Bioinformatics 28:1647-1649.

Klingenberg, C. P., and L. R. Monteiro. 2005. Distances and directions in multidimensional shape spaces: implications for morphometric applications. Syst. Biol. 54:678-688.

Kuchta, S. R., and E. I. Svensson. 2014. Predator-mediated natural selection on the wings of the damselfly Calopteryx splendens: Differences in selection among trait types. Am. Nat. 184:91-109.

Lampert, K. P., C. Schmidt, P. Fischer, J. N. Volff, C. Hoffmann, J. Muck, M. J. Lohse, M. J. Ryan, and M. Schartl. 2010. Determination of onset of sexual maturation and mating behavior by melanocortin receptor 4 polymorphisms. Curr. Biol. 20:1729-1734.

Lande, R. 1980. Sexual dimorphism, sexual selection, and adaptation in polygenic characters. Evolution (N. Y). 34:292-305.

Lande, R., and S. Arnold. 1983. The measurement of selection on correlated characters. Evolution (N. Y). $37: 1210-1226$.

Langerhans, R. B. 2009. Trade-off between steady and unsteady swimming underlies predator-driven divergence in Gambusia affinis. J. Evol. Biol. 22:1057-1075.

Langerhans, R.B., and Reznick, D.N. (2010). "Ecology and Evolution of Swimming Performance in Fishes: Predicting Evolution with Biomechanics," in Fish Locomotion: An Etho-ecological Perspective, eds. P. Domenici \& B.G. Kapoor. (Enfield: Science Publishers), 200-248.

Langerhans, R.B., and Reznick, D.N. (2010). "Ecology and Evolution of Swimming Performance in Fishes: Predicting Evolution with Biomechanics," in Fish Locomotion: An Etho-ecological Perspective, eds. P. Domenici \& B.G. Kapoor. (Enfield: Science Publishers), 200-248.

Lewis, Z., N. Wedell, and J. Hunt. 2011. Evidence for strong intralocus sexual conflict in the indian meal moth, Plodia interpunctella. Evolution (N. Y). 65:2085-2097.

Liotta, M. N., J. K. Abbott, O. Rios-Cardenas, and M. R. Morris. 2019. Tactical dimorphism: the interplay between body shape and mating behaviour in the swordtail Xiphophorus multilineatus (Cyprinodontiformes: Poeciliidae). Biol. J. Linn. Soc. 127:337-350.

Long, T. A., and W. R. Rice. 2007. Adult locomotory activity mediates intralocus sexual conflict in a laboratory-adapted population of Drosophila melanogaster. Proc. R. Soc. B Biol. Sci. 274:3105-3112.

Luo, J., M. Sanetra, M. Schartl, and A. Meyer. 2005. Strong reproductive skew among males in the multiply mated swordtail Xiphophorus multilineatus (Teleostei). J. Hered. 96:346-355.

Lyons, S. M., D. Goedert, and M. R. Morris. 2014. Male-trait-specific variation in female mate preferences. Anim. Behav. 87:39-44. Elsevier Ltd.

MacLaren, R. D., W. J. Rowland, and N. Morgan. 2004. Female preferences for sailfin and body size in the sailfin molly, Poecilia latipinna. Ethology 110:363-379. 
Mank, J. E. 2009. Sex chromosomes and the evolution of sexual dimorphism: lessons from the genome. Am. Nat. 173:141-150.

Meffe, G.K., and F. F. Snelson Jr. 1989. Ecology and evolution of livebearing fishes. Prentice Hall, Englewood Cliffs, N.J.

Mitteroecker, P., and F. Bookstein. 2011. Linear discrimination, ordination, and the visualization of selection gradients in modern morphometrics. Evol. Biol. 38:100-114.

Moczek, A. P., and D. J. Emlen. 2000. Male horn dimorphism in the scarab beetle, Onthophagus taurus: Do alternative reproductive tactics favour alternative phenotypes? Anim. Behav. 59:459-466.

Moretz, J. A., and M. R. Morris. 2003. Evolutionarily labile responses to a signal of aggressive intent. Proc. R. Soc. B Biol. Sci. 270:2271-2277.

Morris, M. R., R. J. Friebertshauser, O. Rios-Cardenas, M. N. Liotta, and J. K. Abbott. 2016. The potential for disruptive selection on growth rates across genetically influenced alternative reproductive tactics. Evol. Ecol. 30:519-533.

Morris, M. R., D. Goedert, J. K. Abbott, D. M. Robinson, and O. Rios-Cardenas. 2013. Intralocus tactical conflict and the evolution of alternative reproductive tactics. Pp. 447-478 in J. H. Brockmann, T. Roper, M. Naguib, J. Mitani, L. Simmons, ed. Advances in the Study of Behavior. Academic Press, USA.

Morris, M., J. Moretz, K. Farley, and P. Nicoletto. 2005. The role of sexual selection in the loss of sexually selected traits in the swordtail fish. Anim. Behav. 69:1415-1424.

Morris, M. R., O. Rios-Cardenas, and J. Brewer. 2010. Variation in mating preference within a wild population influences the mating success of alternative mating strategies. Anim. Behav. 79:673-678.

Morris, M. R., M. S. Tudor, and N. S. Dubois. 2007. Sexually selected signal attracted females before deterring aggression in rival males. Anim. Behav. 74:1189-1197.

Oettler, J., M. Suefuji, and J. Heinze. 2010. The evolution of alternative reproductive tactics in male cardiocondyla ants. Evolution (N. Y). 64:3310-3317.

Oliveria, Rui F., Taborsky, Michael, Brockmann, H. J. 2008. Alternative Reproductive Tactics - An Integrative Approach. Cambridge University Press, New York.

Oufiero, C. E., K. Jugo, and T. Garland. 2014a. Swimming with a sword: Tail beat kinematics in relation to sword length in Xiphophorus. Funct. Ecol. 28:924-932.

Oufiero, C. E., R. W. Meredith, K. N. Jugo, P. Tran, M. A. Chappell, M. S. Springer, D. N. Reznick, and T. Garland. 2014b. The evolution of the sexually selected sword in Xiphophorus does not compromise aerobic locomotor performance. Evolution (N. Y). 68:1806-1823.

Pennell, T. M., L. Holman, E. H. Morrow, and J. Field. 2018. Building a new research framework for social evolution: intralocus caste antagonism. Biol. Rev. 44.

Pike, K. N., J. L. Tomkins, and B. A. Buzatto. 2017. Mixed evidence for the erosion of intertactical genetic correlations through intralocus tactical conflict. J. Evol. Biol. 30:1195-1204.

Piñeros, V. J., O. Rios-Cardenas, C. Gutiérrez-Rodríguez, and L. Mendoza-Cuenca. 2015. Morphological Differentiation in the Damselfish Abudefduf saxatilis Along the Mexican Atlantic Coast is Associated with Environmental Factors and High Connectivity. Evol. Biol. 42:235-249.

R Core Team 2019. R: A language and environment for statistical computing. R Foundation for Statistical Computing, Vienna, Austria. URL https://www.R-project.org/.

Radwan, J., and M. Klimas. 2001. Male dimorphism in the bulb mite, Rhizoglyphus robini: Fighters survive better. Ethol. Ecol. Evol. 13:69-79. 
Rauchenberger, M., K. D. Kallman, and D. C. Morizot. 1990. Monophyly and geography of the Rio Panuco basin swordtails (Genus Xiphophorus) with descriptions of four new species. Am. Museum Novit. 2975:1-41.

Rice, W. R. 1984. Sex chromosomes and the evolution of sexual dimorphism. Evolution (N. Y). 38:735-742.

Rios-Cardenas, O., L. Bono, and M. R. Morris. 2018. Frequency-dependent selection and fluctuations around an equilibrium for alternative reproductive tactics in a swordtail. Anim. Behav. 140:19-28.

Rios-Cardenas, O., and M. R. Morris. 2011. Precopulatory sexual selection. Pp. 187-196 in J. P. Evans, A. Pilastro, I. Schlupp, ed. Ecology and evolution of poeciliid fishes. Univeristy of Chicago Press, Chicago.

Rios-Cardenas, O., M. S. Tudor, and M. R. Morris. 2007. Female preference variation has implications for the maintenance of an alternative mating strategy in a swordtail fish. Anim. Behav. 74:633-640.

Rohlf, F. J. 1999. Shape statistics: procrustes superimpositions and tangent spaces. J. Classif. 16:197-223.

Rohlf, F. J. 2015. The tps series of software. Hystrix 26:1-4.

Rohlf, F. J., and D. Slice. 1990. Extensions of the procrustes method for the optimal superimposition of landmarks. Syst. Zool. 39:40.

Rosenthal, G. G., W. E. Wagner, and M. J. Ryan. 2002. Secondary reduction of preference for the sword ornament in the pygmy swordtail Xiphophorus nigrensis (Pisces: Poeciliidae). Anim. Behav. 63:37-45.

Rousset, F. 2008. GENEPOP'007: A complete re-implementation of the GENEPOP software for Windows and Linux. Mol. Ecol. Resour. 8:103-106.

Royle, N. J., N. B. Metcalfe, and J. Lindström. 2006. Sexual selection, growth compensation and fast-start swimming performance in Green Swordtails, Xiphophorus helleri. Funct. Ecol. 20:662-669.

Ryan, M. J., and B. A. Causey. 1989. "Alternative" mating behavior in the swordtails Xiphophorus nigrensis and Xiphophorus pygmaeus (Pisces: Poeciliidae). Behav. Ecol. Sociobiol. 24:341-348.

Sato, T., M. Hirose, M. Taborsky, and S. Kimura. 2004. Size-dependent male alternative reproductive tactics in the shell-brooding cichlid fish Lamprologus callipterus in Lake Tanganyika. Ethology 110:49-62.

Schneider, C. A., W. S. Rasband, and K. W. Eliceiri. 2012. NIH Image to ImageJ: 25 years of image analysis. Nat. Methods 9:671-675.

Seckinger, J., H. Brinkmann, and A. Meyer. 2002. Microsatellites in the genus Xiphophorus, developed in Xiphophorus montezumae. Mol. Ecol. Notes 2:4-6.

Stinchcombe, J. R., A. F. Agrawal, P. A. Hohenlohe, S. J. Arnold, and M. W. Blows. 2008. Estimating nonlinear selection gradients using quadratic regression coefficients: Double or nothing? Evolution (N. Y). 62:2435-2440.

Taborsky, M. 2001. The evolution of bourgeois, parasitic, and cooperative reproductive behaviors in fishes. J. Hered. 92:100-110.

Taborsky, M., and H. Brockmann. 2010. Alternative reproductive tactics and life history phenotypes. Pp. 537- 586 in P. Kappeler, ed. Animal behaviour: Evolution and mechanisms.

Van Oosterhout, C., W. F. Hutchinson, D. P. M. Wills, and P. Shipley. 2004. MICRO-CHECKER: Software for identifying and correcting genotyping errors in microsatellite data. Mol. Ecol. Notes 4:535-538.

Webb, P. W. 1984. Body Form, Locomotion and Foraging in Aquatic Vertebrates. Am. Zool. 24:107-120.

Webb, P. W. 1994. The biology of fish swimming. Pp. 45-62 in J. M. V. Maddock, L., Bone, Q., Rayner, ed. Mechanics and Physiology of Animal Swimming. Cambridge University Press. 
Weinstein, M., M. N. Liotta, A. Solitt, A. Hunt, J. K. Abbott, O. Rios-Cardenas, and M. R. Morris. 2019. Selection on growth rates via a trade-off between survival to sexual maturity and longevity in the swordtail fish Xiphophorus multilineatus. Evol. Ecol. 33:549-566.

West-Eberhard, M. J. 1986. Alternative adaptations, speciation, and phylogeny (A Review). Proc. Natl. Acad. Sci. U. S. A. 83:1388-1392.

Zelditch M.L., D.L. Swiderski, H.D. Sheets. 2012. Geometric morphometrics for biologists: a primer. Academic Press, San Diego, CA.

Zimmerer, E. J., and K. D. Kallman. 1989. Genetic basis for alternative reproductive tactics in the pygmy swordtail, Xiphophorus nigrensis. Evolution (N. Y). 43:1298.

Zimmerer, E. J., and K. D. Kallman. 1988. The inheritance of vertical barring (aggression and appeasement signals) in the pygmy swordtail, Xiphophorus nigrensis (Poeciliidae, Teleostei). Copeia 1988:299-307.

\section{Figure Legends}

Figure 1. Courter (A) and sneaker (B) males of Xiphophorus multilineatus. a, vertical body bars; a*, no vertical body bars present on this sneaker male; b, the sword, extension of the ventral caudal fin rays; C) Sampling for DNA and morphology took place in a side stream of the Río Tambaque located just outside of Aquismón, San Luis Potosí, Mexico. D) Red filled circles = landmarks; red open circles = semi-landmarks; blue line = semi-landmark markers or linear measurements; numbers correspond to landmark locations (see Material and Methods). (A, B photos by Luke Weinstein; C by Melissa Liotta)

Figure 2. Principal components analysis of the variation in body shape along the first two principal component $(\mathrm{PC})$ axes. Orange triangles $=$ sneaker males; Blue circles $=$ courter males. Wire-frame models representing the extreme minimal and maximal changes in shape along PC1 and PC2 are shown (red $=$ consensus shape, i.e. average body shape of all males; black = shape change, i.e. the change in body shape from the average body shape). Small filled ellipses are the $95 \%$ confidence regions around the respective group centroids.

Figure 3. Selection differentials for each unidimensional trait and trait distribution by ART. Solid line is the differential $(s)$ and shaded regions are the $95 \%$ confidence intervals. Vertical dashed lines show the mean trait value for each ART. Histograms show the distribution of the trait values. Large purple asterisks indicate a significant difference between the slopes. Blue and orange asterisks indicate a significant slope. Orange $=$ sneaker males, blue $=$ courter males. ${ }^{*} \mathrm{P}[?] \quad 0.05,{ }^{* *} \mathrm{P}$ [?] $0.01{ }^{* * *} \mathrm{P}[?] 0.001$

Figure 4. Selection differentials for each geomorphometric trait and trait distribution by ART. Solid line is the differential $(s)$ and shaded regions are the $95 \%$ confidence intervals. Vertical dashed lines show the mean trait value for each ART. Histograms show the distribution of the trait values. Large purple asterisks indicate a significant difference between the slopes. Blue and orange asterisks indicate a significant slope. Wireframes as explained in Figure 2. Orange $=$ sneaker males, blue $=$ courter males. + Average slope between the ARTs is significant, we did not perform subsequent separate models in these cases because the interaction between the trait and ART was not significant (see Table $2 \mathrm{~A}$ ). ${ }^{*} \mathrm{P}[?] \quad 0.05,{ }^{* *} \mathrm{P}[?] 0.01{ }^{* * *} \mathrm{P}[?]$ 0.001 


\begin{tabular}{|c|c|c|c|c|c|c|c|}
\hline \multirow{2}{*}{ A } & \multicolumn{3}{|c|}{ Differential $(s)$} & \multirow[b]{2}{*}{ Courter (s) } & \multirow[b]{2}{*}{$\mathrm{P}^{*}$} & \multirow[b]{2}{*}{ Sneaker (s) } & \multirow[b]{2}{*}{$\mathrm{P}^{*}$} \\
\hline & SS & $\mathrm{F}_{3,76}$ & $\mathrm{P}$ & & & & \\
\hline Standard Length (size) & 18.05 & 7.83 & 0.01 & 1.38 & 0.02 & -0.53 & 0.13 \\
\hline ART:Standard Length & 10.49 & 4.55 & 0.02 & & & & \\
\hline Body Depth & 13.55 & 5.7 & 0.02 & 1.3 & 0.04 & -0.18 & 0.32 \\
\hline ART:Body Depth & 7.29 & 3.07 & 0.05 & & & & \\
\hline Sword Length & 37.16 & 18.41 & ¡0.0 & 1.58 & 0.0 & -0.64 & 0.03 \\
\hline $\begin{array}{c}\text { ART:Sword Length } \\
\text { B }\end{array}$ & 28.07 & $\begin{array}{c}13.91 \\
\text { Linear }(ß)\end{array}$ & 0.0 & & & & \\
\hline & SS & $\mathrm{F}_{7,72}$ & $\mathrm{P}$ & Courter (B) & $\mathrm{P}^{*}$ & Sneaker ( $($ ) & $\mathrm{P}^{*}$ \\
\hline Standard Length (size) & 8.77 & 4.38 & 0.04 & 2.1 & - & -0.09 & - \\
\hline ART:Standard Length & 4.06 & 2.03 & 0.11 & & & & \\
\hline Body Depth & 5.13 & 2.57 & 0.08 & -1.88 & - & 0.24 & - \\
\hline ART:Body Depth & 4.27 & 2.13 & 0.1 & & & & \\
\hline Sword Length & 28.21 & 14.1 & 0.0 & 1.64 & 0.01 & -0.68 & 0.06 \\
\hline ART:Sword Length & 19.53 & 9.77 & 0.01 & & & & \\
\hline $\mathrm{C}$ & & Quadradic $\left(\mathrm{y}_{\mathrm{ii}}\right)$ & & & & & \\
\hline & SS & $\mathrm{F}_{13,66}$ & $\mathrm{P}$ & Courter $\left(\mathrm{y}_{\mathrm{ii}}\right)$ & & Sneaker $\left(y_{i i}\right)$ & \\
\hline Standard Length (size) & 0.39 & 0.22 & 0.32 & 1.51 & & 0.47 & \\
\hline ART:Standard Length & 0.06 & 0.04 & 0.44 & & & & \\
\hline Body Depth & 0.4 & 0.23 & 0.34 & 1.92 & & 0.71 & \\
\hline ART:Body Depth & 0.1 & 0.06 & 0.42 & & & & \\
\hline Sword Length & 10.06 & 5.74 & 0.05 & 2.39 & & 1.72 & \\
\hline ART:Sword Length & 0.36 & 0.21 & 0.34 & & & & \\
\hline
\end{tabular}

Table 1: Selection analyses on the unidimensional traits. (A) differential selection model with both ARTs, in addition to analyses of ARTs separately for traits that had a significant interaction with ART; (B) linear and (C) quadratic selection models including all traits and interactions with ART. ART = alternative reproductive tactic, $\mathrm{SS}=$ sums of squares, $\mathrm{F}=\mathrm{ANOVA} \mathrm{F}$ statistic, $\mathrm{P}=\mathrm{p}$-value. $\mathrm{P}$-values calculated using a resampling procedure (Lewis et al. 2011). Significant P-values highlighted in bold. P* were calculated from separate analyses by ART in cases where interactions were significant. 


\begin{tabular}{|c|c|c|c|c|c|c|c|}
\hline$A$ & & Differential $(s)$ & & & & & \\
\hline & SS & $\mathrm{F}_{3,76}$ & $\mathrm{P}$ & Courter (s) & $\mathrm{P}^{*}$ & Sneaker (s) & $\mathrm{P}^{*}$ \\
\hline Centroid Size & 21.26 & 9.35 & 0.01 & 1.24 & - & -0.27 & - \\
\hline ART:Centroid size & 5.19 & 2.28 & 0.07 & & & & \\
\hline Body shape (PC1) & 17.39 & 7.48 & 0.01 & 1.08 & 0.02 & -0.09 & 0.33 \\
\hline $\mathrm{ART}: \mathrm{PC} 1$ & 11.3 & 4.86 & 0.02 & & & & \\
\hline Body shape (PC2) & 3.55 & 1.41 & 0.11 & 0.39 & - & 0.04 & - \\
\hline ART:PC2 & 1.97 & 0.78 & 0.17 & & & & \\
\hline Body shape (PC3) & 3.16 & 1.31 & 0.11 & 0.27 & 0.17 & -0.48 & 0.01 \\
\hline ART:PC3 & 10.63 & 4.4 & 0.02 & & & & \\
\hline Body shape (PC4) & 0.0 & 0 & 0.49 & -0.0 & - & 0.2 & - \\
\hline ART:PC4 & 0.74 & 0.29 & 0.29 & & & & \\
\hline Sword length & 37.16 & 18.41 & ¡0.0 & 1.58 & 0.0 & -0.64 & 0.03 \\
\hline ART:Sword & 28.07 & 13.91 & 0.0 & & & & \\
\hline B & & Directional ( $(\beta)$ & & & & & \\
\hline & SS & $\mathrm{F}_{13,66}$ & $\mathrm{P}$ & Courter $(\beta)$ & $\mathrm{P}^{*}$ & Sneaker $(\beta)$ & $\mathrm{P}^{*}$ \\
\hline Centroid Size & 11.01 & 6.21 & 0.03 & 1.14 & - & 0.42 & - \\
\hline ART:Centroid size & 0.74 & 0.42 & 0.29 & & & & \\
\hline Body shape (PC1) & 5.23 & 2.95 & 0.07 & -0.93 & - & 0.03 & - \\
\hline ART:PC1 & 3.77 & 2.13 & 0.11 & & & & \\
\hline Body shape (PC2) & 14.72 & 8.3 & 0.02 & 0.86 & 0.04 & 0.09 & 0.29 \\
\hline ART:PC2 & 7.54 & 4.26 & 0.05 & & & & \\
\hline Body shape (PC3) & 9.55 & 5.39 & 0.02 & 0.5 & 0.05 & -0.45 & 0.01 \\
\hline ART:PC3 & 15.53 & 8.76 & 0.01 & & & & \\
\hline Body shape (PC4) & 1.79 & 1.01 & 0.19 & -0.26 & - & 0.18 & - \\
\hline ART:PC4 & 3.36 & 1.9 & 0.13 & & & & \\
\hline Sword length & 29.55 & 16.67 & 0.0 & 1.95 & 0.01 & -0.74 & 0.04 \\
\hline ART:Sword & 23.84 & 13.45 & 0.0 & & & & \\
\hline $\mathrm{C}$ & & Quadratic $\left(y_{i i}\right)$ & & & & & \\
\hline & SS & $\mathrm{F}_{25,54}$ & $\mathrm{P}$ & Courter $\left(\mathrm{y}_{\mathrm{ii}}\right)$ & & Sneaker $\left(y_{i i}\right)$ & \\
\hline Centroid Size & 14.31 & 8.93 & 0.02 & 3.32 & & 2.37 & \\
\hline ART:Centroid size & 0.05 & 0.03 & 0.47 & & & & \\
\hline Body shape (PC1) & 0.14 & 0.09 & 0.37 & 0.33 & & -0.65 & \\
\hline ART:PC1 & 0.74 & 0.46 & 0.29 & & & & \\
\hline Body shape (PC2) & 3.07 & 1.91 & 0.11 & -0.49 & & -0.17 & \\
\hline ART:PC2 & 0.61 & 0.38 & 0.31 & & & & \\
\hline Body shape (PC3) & 1.49 & 0.93 & 0.19 & 0.18 & & 0.97 & \\
\hline ART:PC3 & 2.9 & 1.81 & 0.14 & & & & \\
\hline Body shape (PC4) & 0.08 & 0.05 & 0.39 & 0.14 & & -0.06 & \\
\hline ART:PC4 & 0.13 & 0.08 & 0.4 & & & & \\
\hline Sword length & 1.05 & 0.66 & 0.24 & 1.05 & & 1.03 & \\
\hline ART:Sword & 0.0 & 0.0 & 0.49 & & & & \\
\hline
\end{tabular}

Table 2: Selection analyses for the geomorphometric traits, including sword length. (A) differential selection model with both ARTs, in addition to analyses of ARTs separately for traits that had a significant interaction with ART; (B) linear and (C) quadratic selection models including all traits and interactions with ART; separate analyses by ART if interaction for trait was significant. ART = alternative reproductive tactic, SS $=$ sums of squares, $F=A N O V A F$ statistic, $P=$ p-value, $D F=$ degrees of freedom. $P$-values calculated using a resampling procedure (Lewis et al. 2011). Significant P-values highlighted in bold. $P^{*}$ were calculated from separate analyses by ART in cases where interactions were significant. 

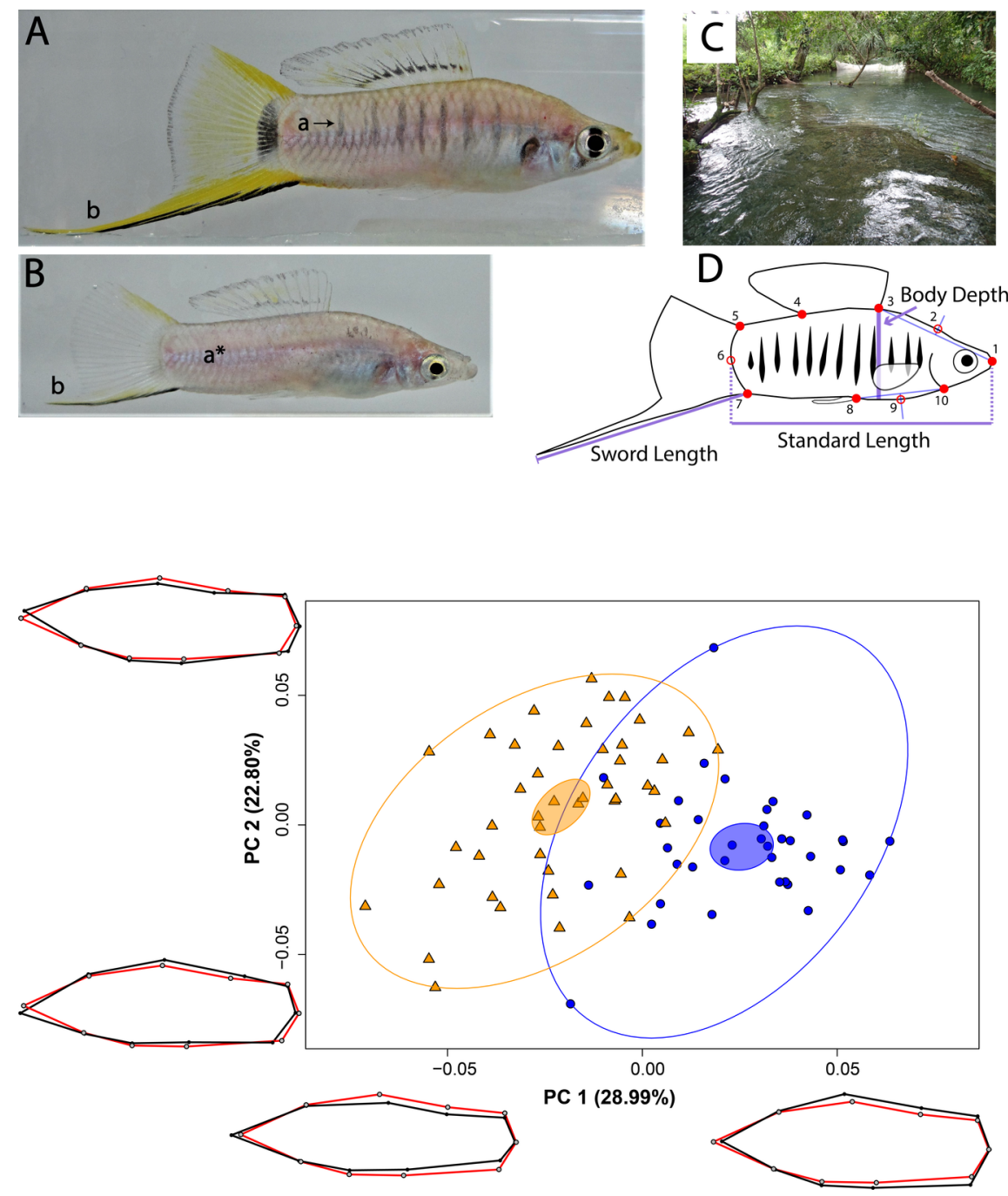

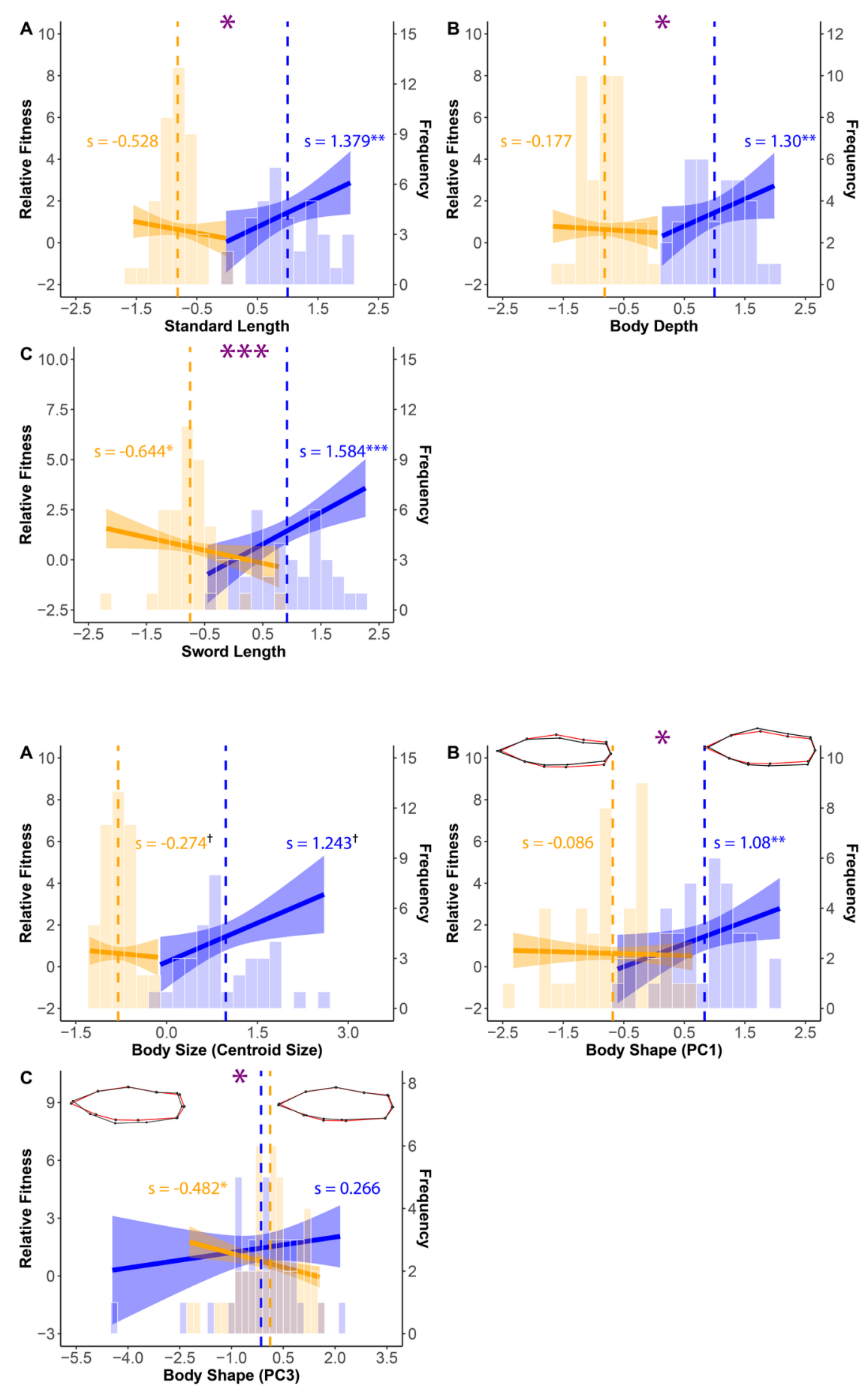

\section{APPENDIX}

\section{Microsatellite Genotyping}

Primer stock was made to include a $2 \mu \mathrm{M}$ concentration of each primer (forward and reverse) for each multiplex, except for KonD6 in MIX1, which had a $1 \mu \mathrm{M}$ concentration due to its intense peaks exhibited in test plates. The PCR cocktail for each sample consisted of a final volume of $10 \mu \mathrm{l}$ and the following: 2.55 
$\mu \mathrm{l}$ of RNAse-Free Water (Type-it Microsatellite PCR kit, Qiagen, Hilden, Germany), $5.0 \mu \mathrm{l}$ of 2x Type-it Microsatellite PCR Master Mix (Qiagen, Hilden, Germany, final concentration 0.06x), $1.45 \mu$ l of the primer stock (final concentration $0.29 \mu \mathrm{M}$ ), and $1 \mu \mathrm{l}$ of DNA.

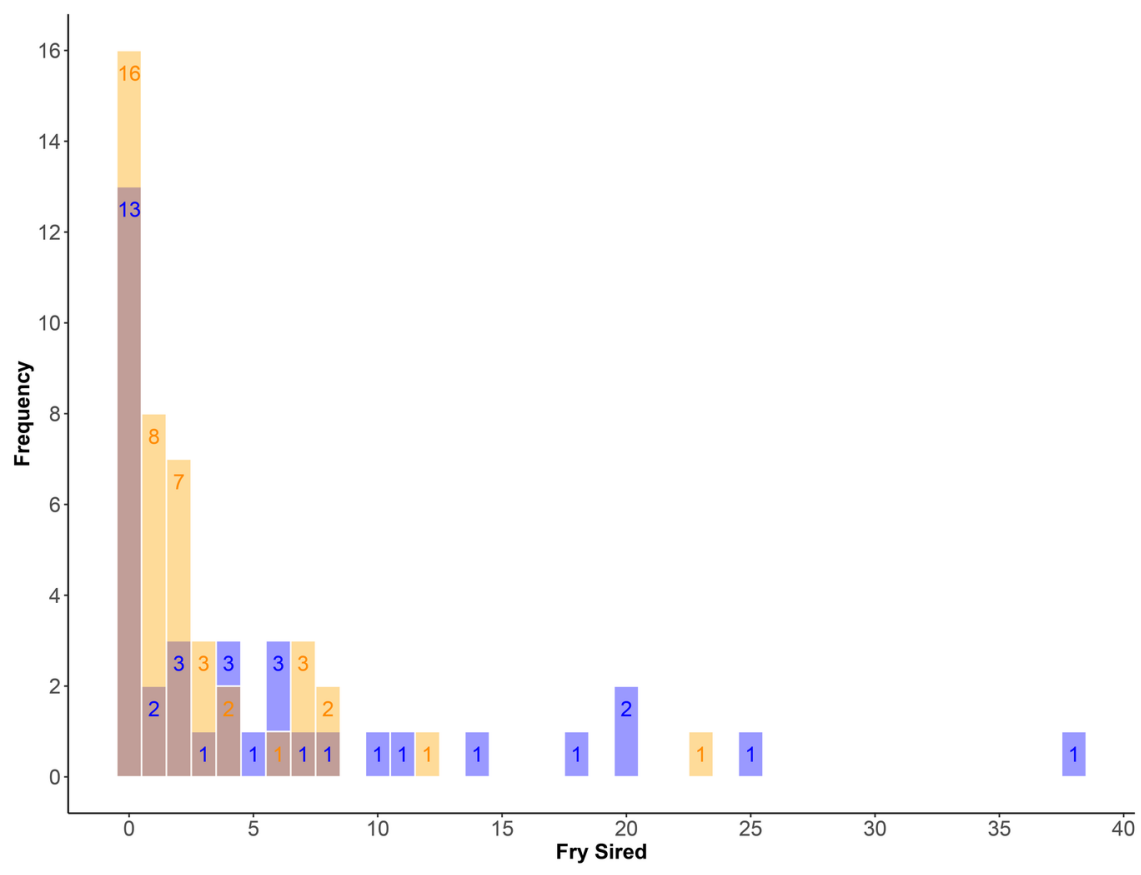

Figure Appendix 1. Frequency of how many males in each ART (courters = blue, sneakers = orange) sired a certain number of fry. Numbers within each bin correspond to the number of sire
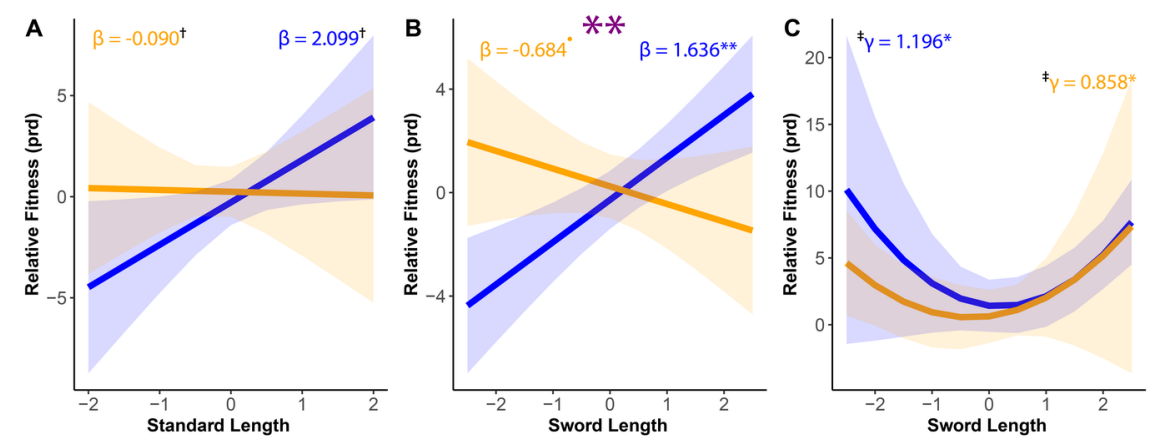
Figure Appendix 2. Marginal effects plots for traits in the unidimensional analysis showing the effect of each response variable on the predicated values (prd) of relative fitness when all other traits are held constant. Solid line is the selection gradient $\left(b\right.$ or $\mathrm{g}_{\mathrm{ii}}$ ) and shaded regions are the $95 \%$ confidence intervals. Large purple asterisks indicate a significant difference between the slopes. Blue and orange asterisks indicate a significant slope. Orange $=$ sneaker males, blue $=$ courter males. + Average slope between the ARTs is significant, we did not perform subsequent separate models in these cases because the interaction between the trait and ART was not significant (see Table 1B-C). ++ Quadratic coefficients visualized here, are on the original scale but are reported in Table 1 multiplied by 2 as suggested by Stinchcombe et al. $2008 .{ }^{*} \mathrm{P}$ $=0.06,{ }^{*} \mathrm{P}[?] \quad 0.05,{ }^{*} \mathrm{P}[?] \quad 0.01 * * * \mathrm{P}[?] 0.001$
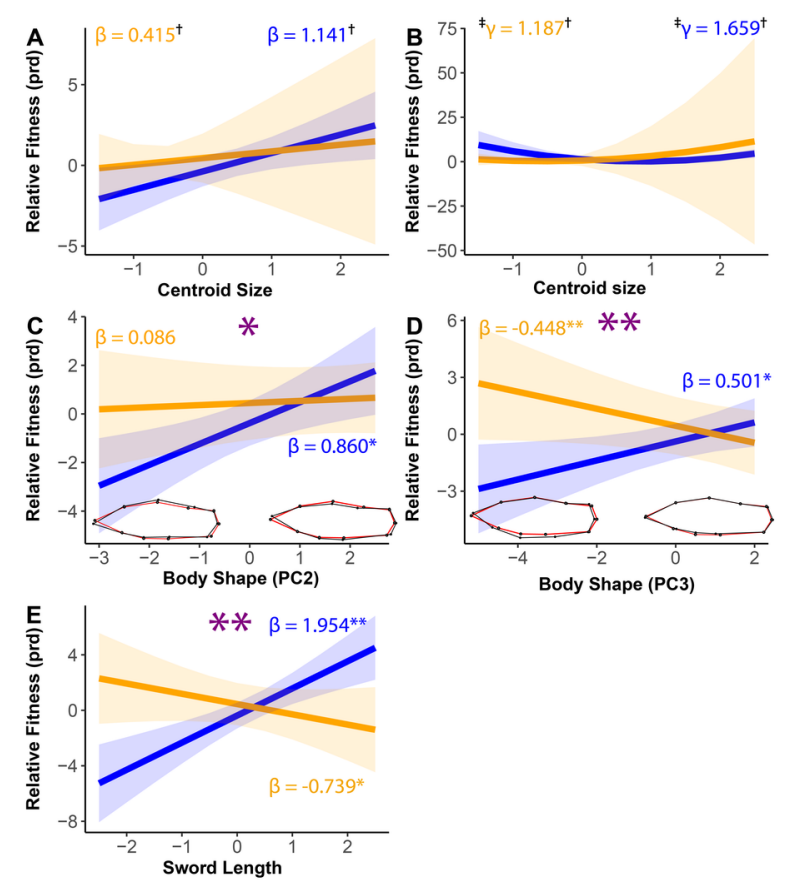

Figure Appendix 3. Marginal effects plots for traits in the geomorphometric analysis showing the effect of each response variable on the predicated values (prd) of relative fitness when all other traits are held constant. Solid line is the selection gradient (b or g) and shaded regions are the $95 \%$ confidence intervals. Large purple asterisks indicate a significant difference between the slopes. Blue and orange asterisks indicate a significant slope. Orange $=$ sneaker males, blue $=$ courter males. Wire-frame models as explained in Figure 2. + Average slope between the ARTs is significant, we did not perform subsequent separate models in these cases because the interaction between the trait and ART were not significant (see Table 2B-C). ++ Quadratic coefficients visualized here, are on the original scale but are reported in Table 2 multiplied by 2 as suggested by Stinchcombe et al. 2008. ${ }^{*} \mathrm{P}=0.06,{ }^{*} \mathrm{P}$ [?] $0.05,{ }^{* *} \mathrm{P}$ [?] $0.01{ }^{* * *} \mathrm{P}[?] 0.001$ 